\title{
Cremenolide, a new 10-member lactone from Trichoderma cremeum with plant growth promotion activity
}

Francesco Vinale ${ }^{1 *}$, Judyta Strakowska ${ }^{2}$, Pierluigi Mazzei ${ }^{3}$, Alessandro Piccolo ${ }^{3}$, Roberta Marra $^{1}$, Nadia Lombardi ${ }^{1}$, Gelsomina Manganiello ${ }^{2}$, Alberto Pascale ${ }^{2}$, Sheridan L. Woo ${ }^{1,2}$ and Matteo Lorito ${ }^{1,2}$

1 Istituto per la Protezione Sostenibile delle Piante, Consiglio Nazionale delle Ricerche, via Università 133, 80055 Portici, Italy

2 Dipartimento di Agraria, Università di Napoli 'Federico II', Portici, 80055 Naples, Italy

3 Centro Interdipartimentale di Spettroscopia di Risonanza Magnetica Nucleare (CERMANU), Università degli Studi di Napoli 'Federico II', Portici, 80055 Naples, Italy

* Author to whom correspondence should be addressed; E-Mail: f.vinale@ipp.cnr.it; Tel.: +39-081-253-9338; Fax: +39-081-253-9339.

\section{Appendix A. Supplementary data}

Figure S1. ${ }^{1} \mathrm{HNMR}$ spectrum $\left(\delta, \mathrm{CDCl}_{3}\right)$ of $\mathbf{1}$.

Figure S2. ${ }^{13} \mathrm{C}$ NMR spectrum $\left(\delta, \mathrm{CDCl}_{3}\right)$ of $\mathbf{1}$.

Figure S3. ${ }^{13} \mathrm{C} D E P T 135 \mathrm{NMR}$ spectrum $\left(\delta, \mathrm{CDCl}_{3}\right)$ of $\mathbf{1}$

Figure S4. ${ }^{1} \mathrm{H}-{ }^{1} \mathrm{H}$ COSY spectrum $\left(\delta, \mathrm{CDCl}_{3}\right)$ of $\mathbf{1}$.

Figure S5. ${ }^{1} \mathrm{H}^{-13} \mathrm{C} \mathrm{HSQC}$ spectrum $\left(\delta, \mathrm{CDCl}_{3}\right)$ of $\mathbf{1}$.

Figure S6. ${ }^{1} \mathrm{H}-{ }^{13} \mathrm{C} \mathrm{HMBC}$ spectrum $\left(\delta, \mathrm{CDCl}_{3}\right)$ of $\mathbf{1}$.

Figure S7. ${ }^{1} \mathrm{H}-{ }^{1} \mathrm{H}$ TOCSY spectrum $\left(\delta, \mathrm{CDCl}_{3}\right)$ of $\mathbf{1}$.

Figure S8. ${ }^{1} \mathrm{H}-{ }^{1} \mathrm{H}$ NOESY spectrum $\left(\delta, \mathrm{CDCl}_{3}\right)$ of $\mathbf{1}$.

Figure S9. Mass spectrum (HRMS) of compound 1.

Figure S10. MS/MS of compound 1.

Figure S11. Determinant TOCSY (A) and NOESY (B) correlations detected for cremenolide (1).

Table $\mathbf{S 1}{ }^{1} \mathrm{H}$ and ${ }^{13} \mathrm{C}$ NMR spectral data of cremenolide (in $\mathrm{CDCl}_{3}$ ). 
Figure S1. ${ }^{1} \mathrm{H}$ NMR spectrum $\left(\delta, \mathrm{CDCl}_{3}\right)$ of $\mathbf{1}$.

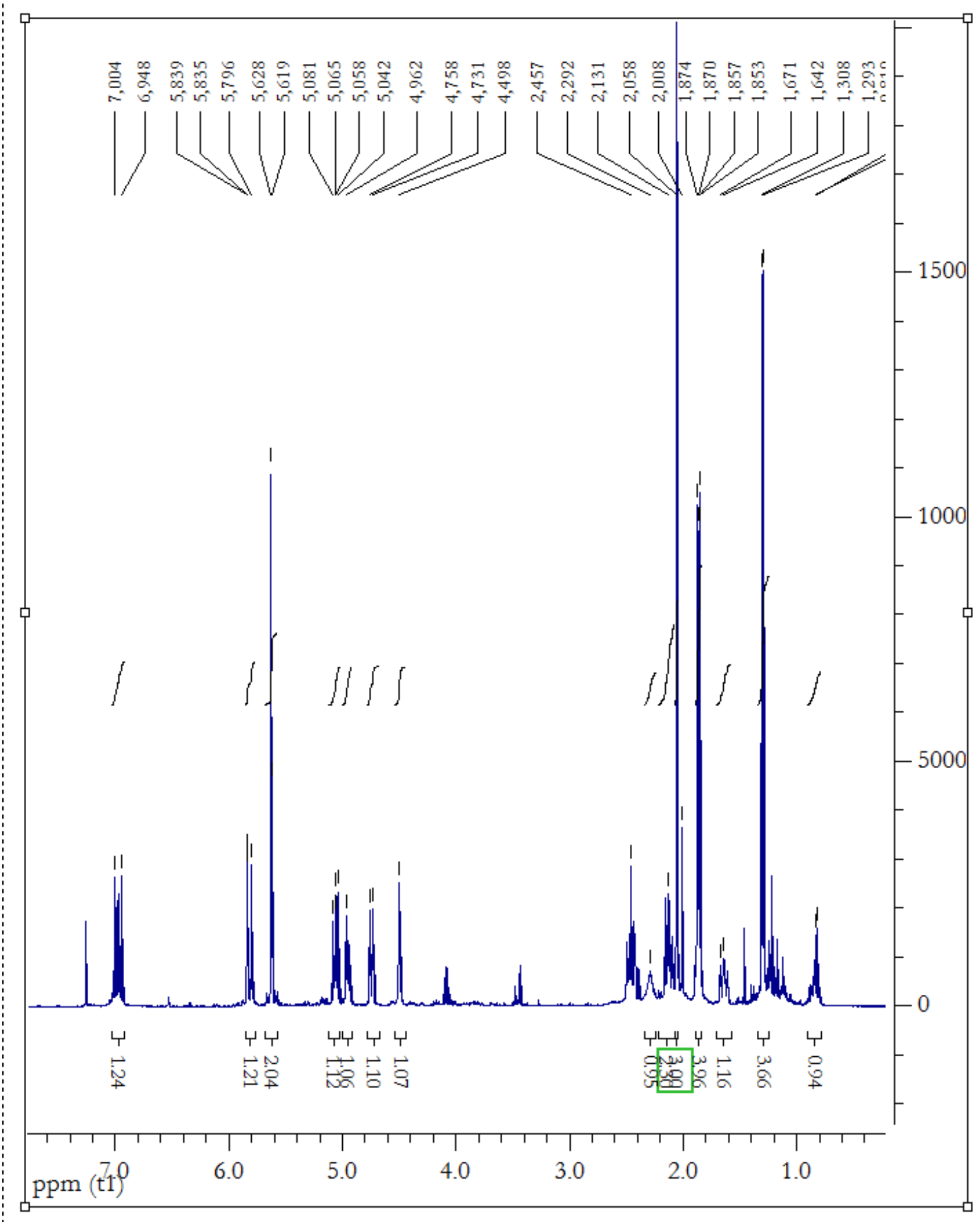


Figure S2. ${ }^{13} \mathrm{C}$ NMR spectrum $\left(\delta, \mathrm{CDCl}_{3}\right)$ of $\mathbf{1}$.

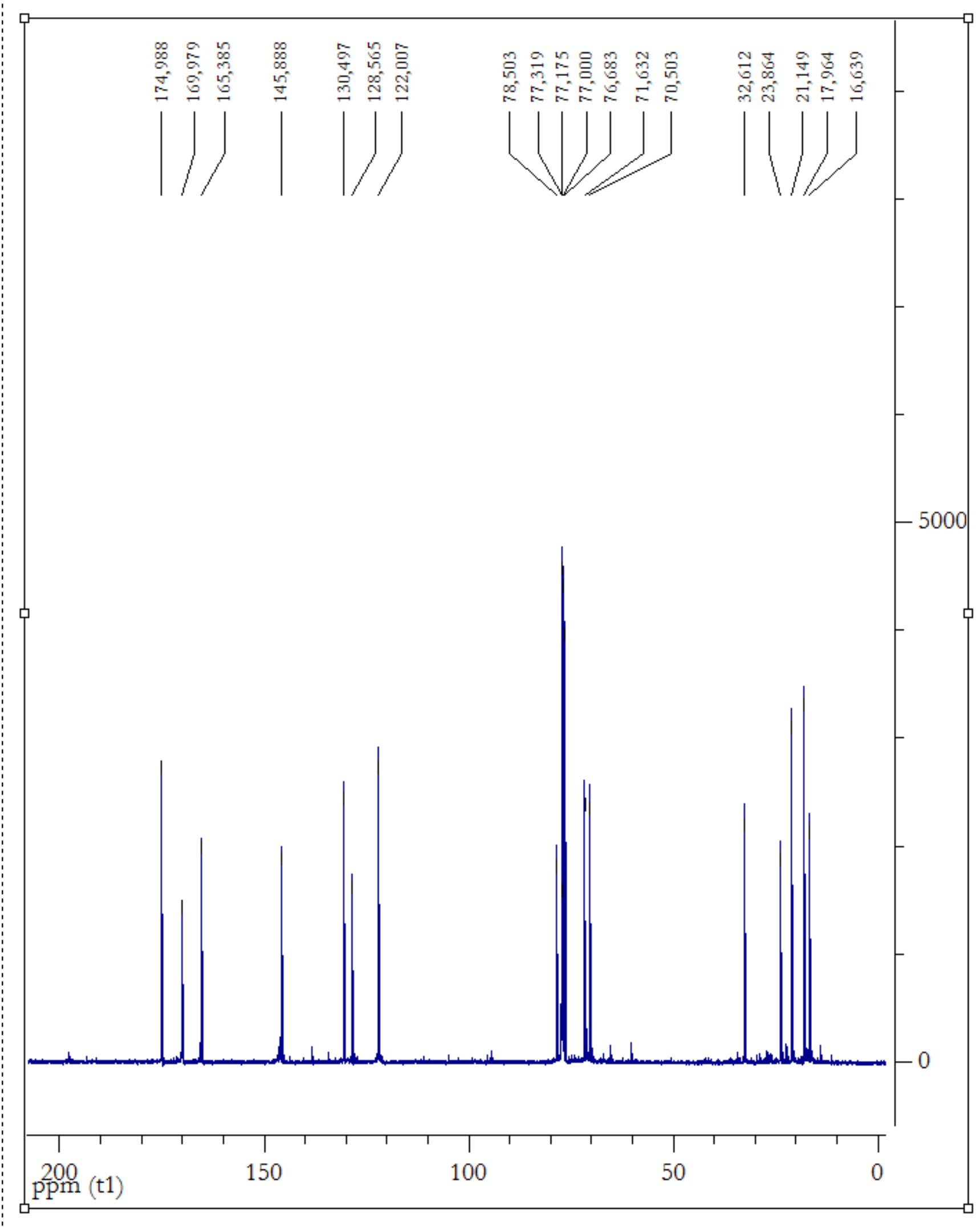


Figure S3. ${ }^{13} \mathrm{C} D E P T 135 \mathrm{NMR}$ spectrum $\left(\delta, \mathrm{CDCl}_{3}\right)$ of 1.

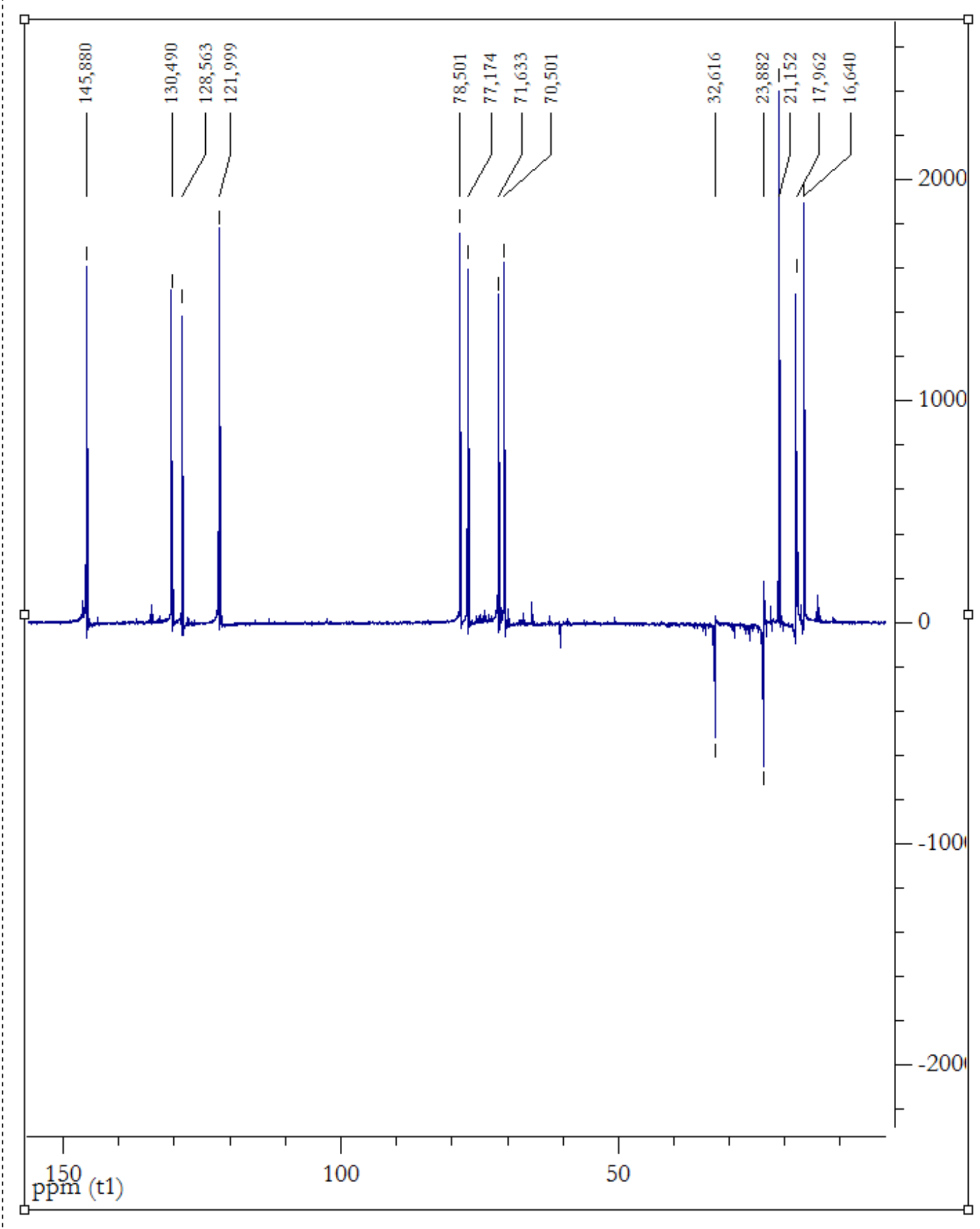


Figure S4. ${ }^{1} \mathrm{H}-{ }^{1} \mathrm{H}$ COSY spectrum $\left(\delta, \mathrm{CDCl}_{3}\right)$ of $\mathbf{1}$.

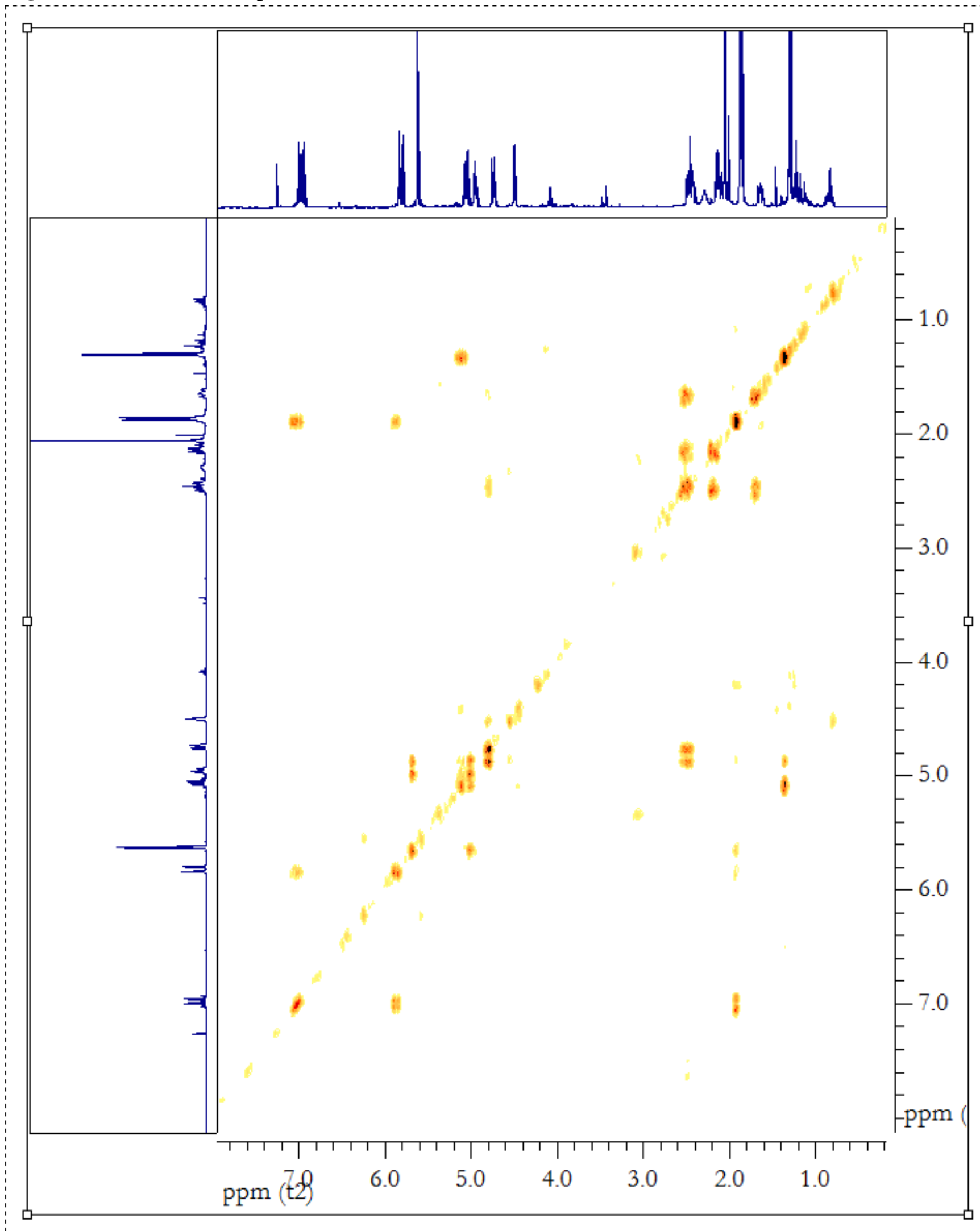


Figure S5. ${ }^{1} \mathrm{H}-{ }^{13} \mathrm{C} \mathrm{HSQC}$ spectrum $\left(\delta, \mathrm{CDCl}_{3}\right)$ of 1.

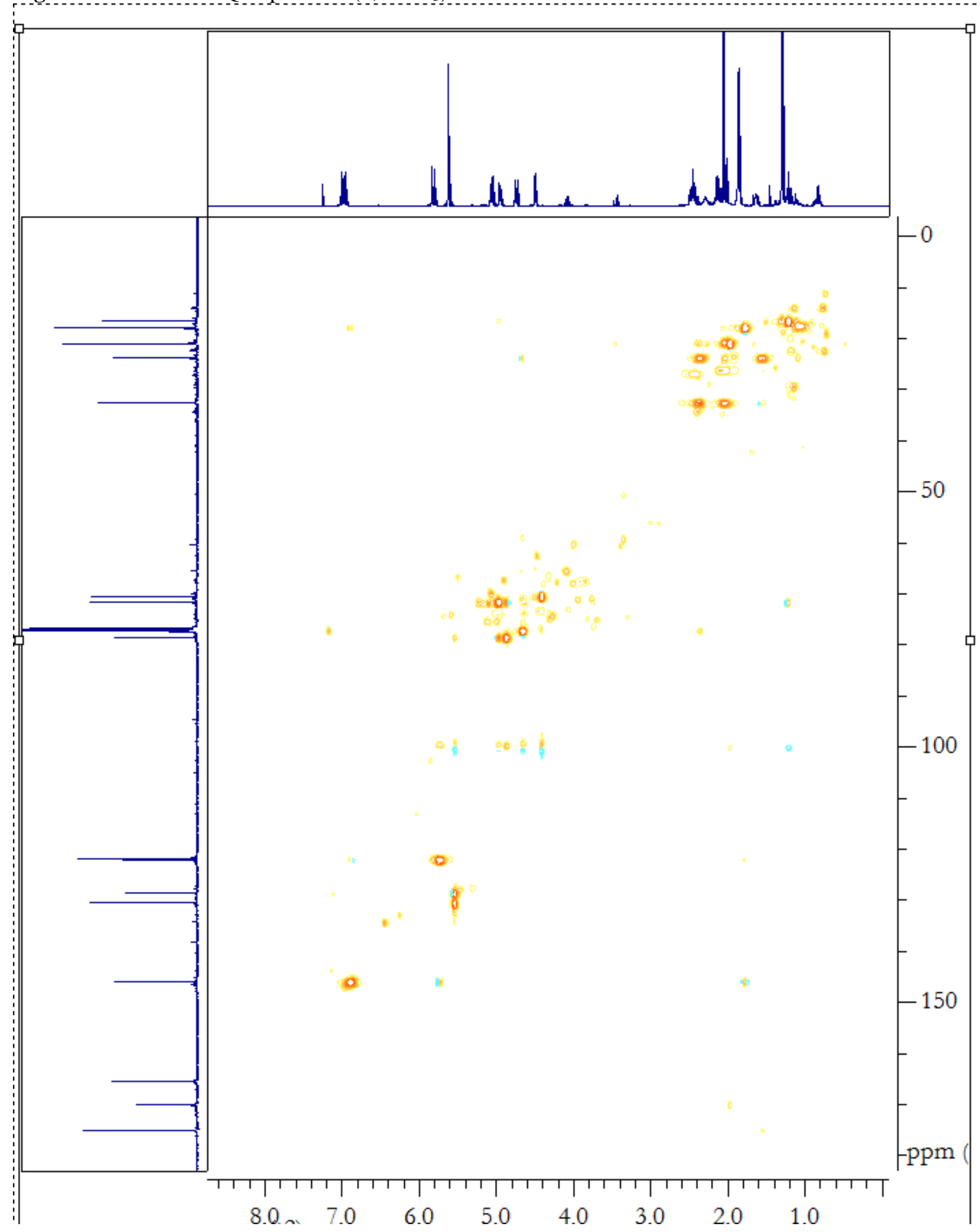


Figure S6. ${ }^{1} \mathrm{H}^{-13} \mathrm{C} \mathrm{HMBC}$ spectrum $\left(\delta, \mathrm{CDCl}_{3}\right)$ of $\mathbf{1}$

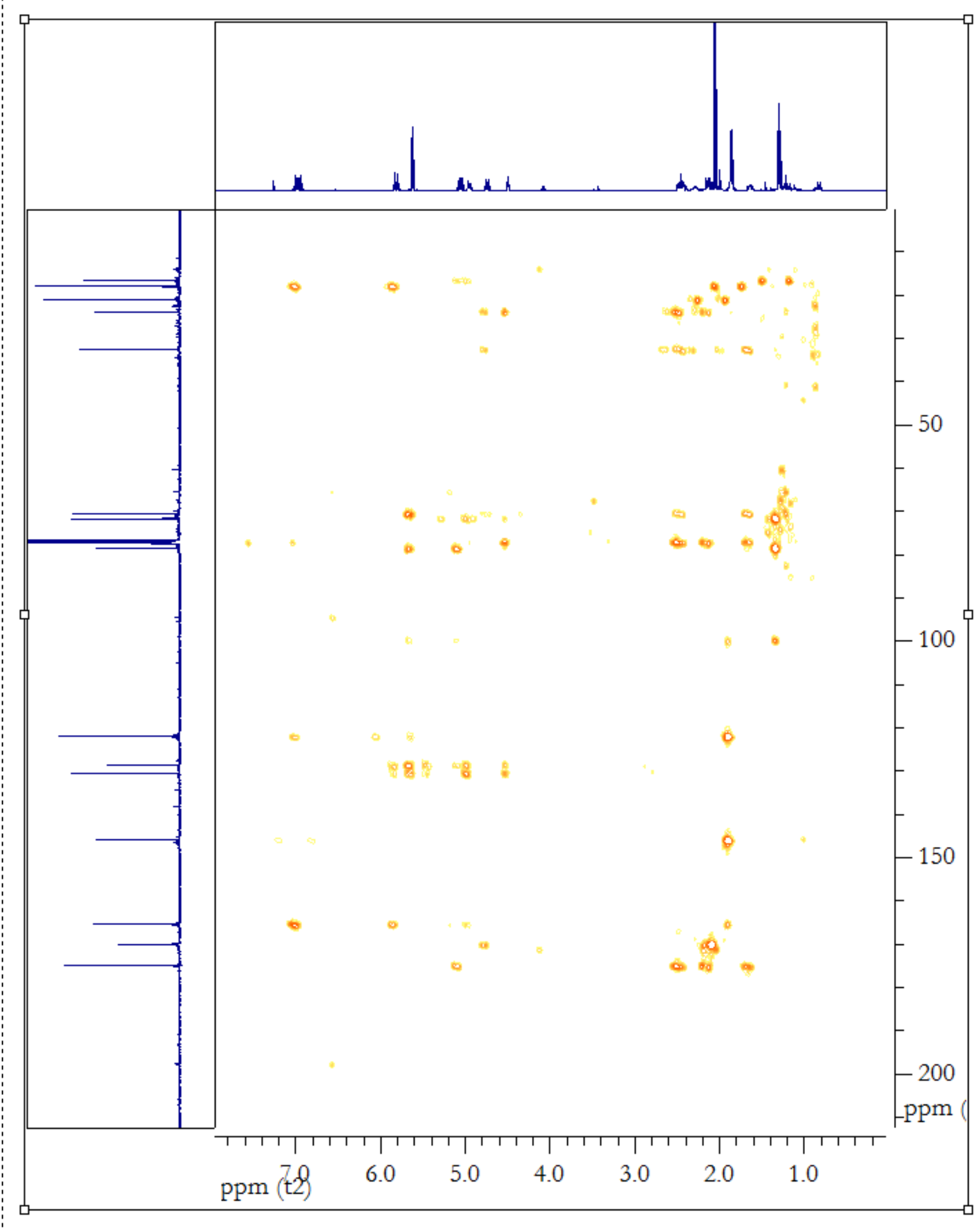


Figure S7. ${ }^{1} \mathrm{H}-{ }^{1} \mathrm{H}$ TOCSY spectrum $\left(\delta, \mathrm{CDCl}_{3}\right)$ of 1.

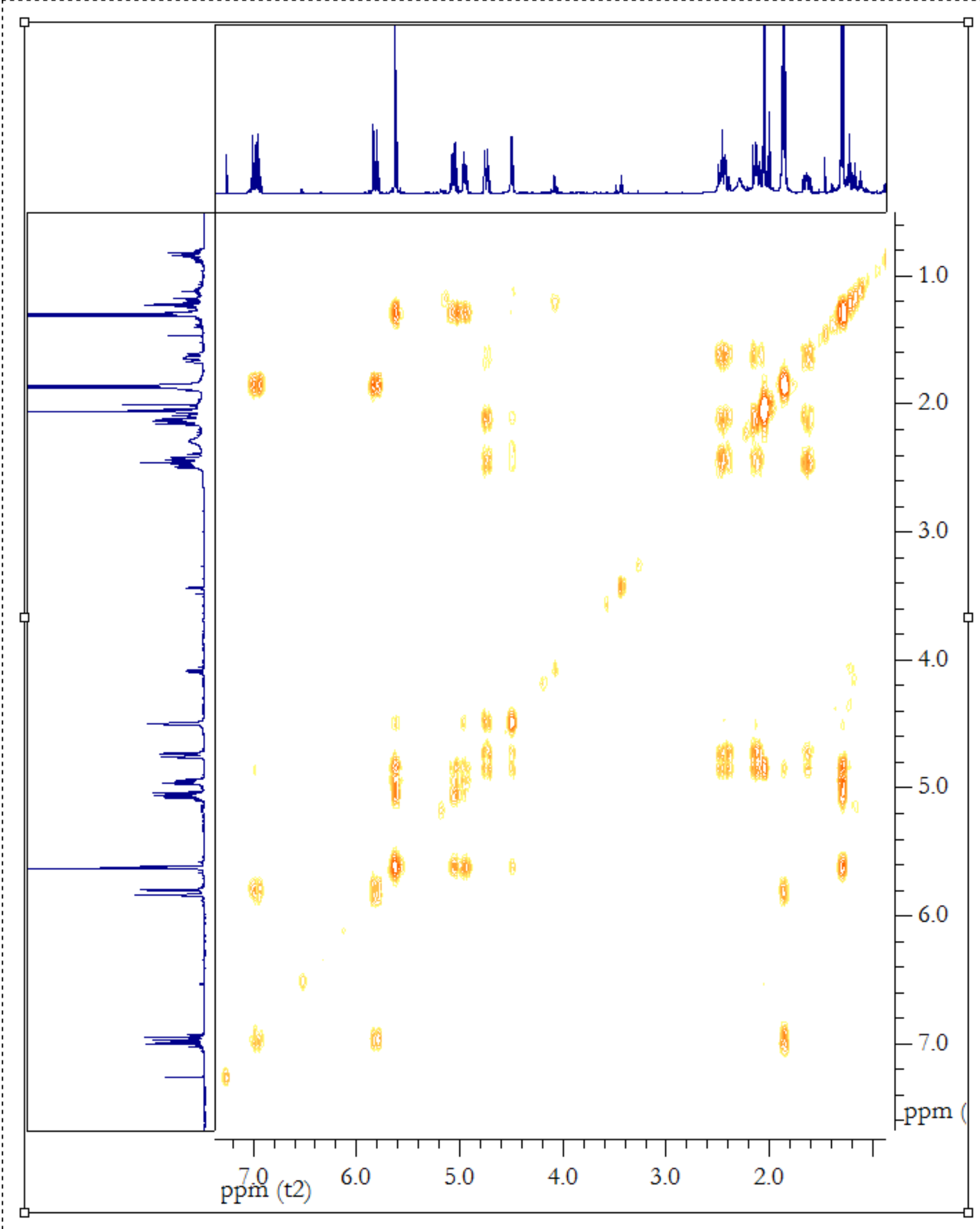


Figure S8. ${ }^{1} \mathrm{H}-{ }^{1} \mathrm{H}$ NOESY spectrum $\left(\delta, \mathrm{CDCl}_{3}\right)$ of 1.

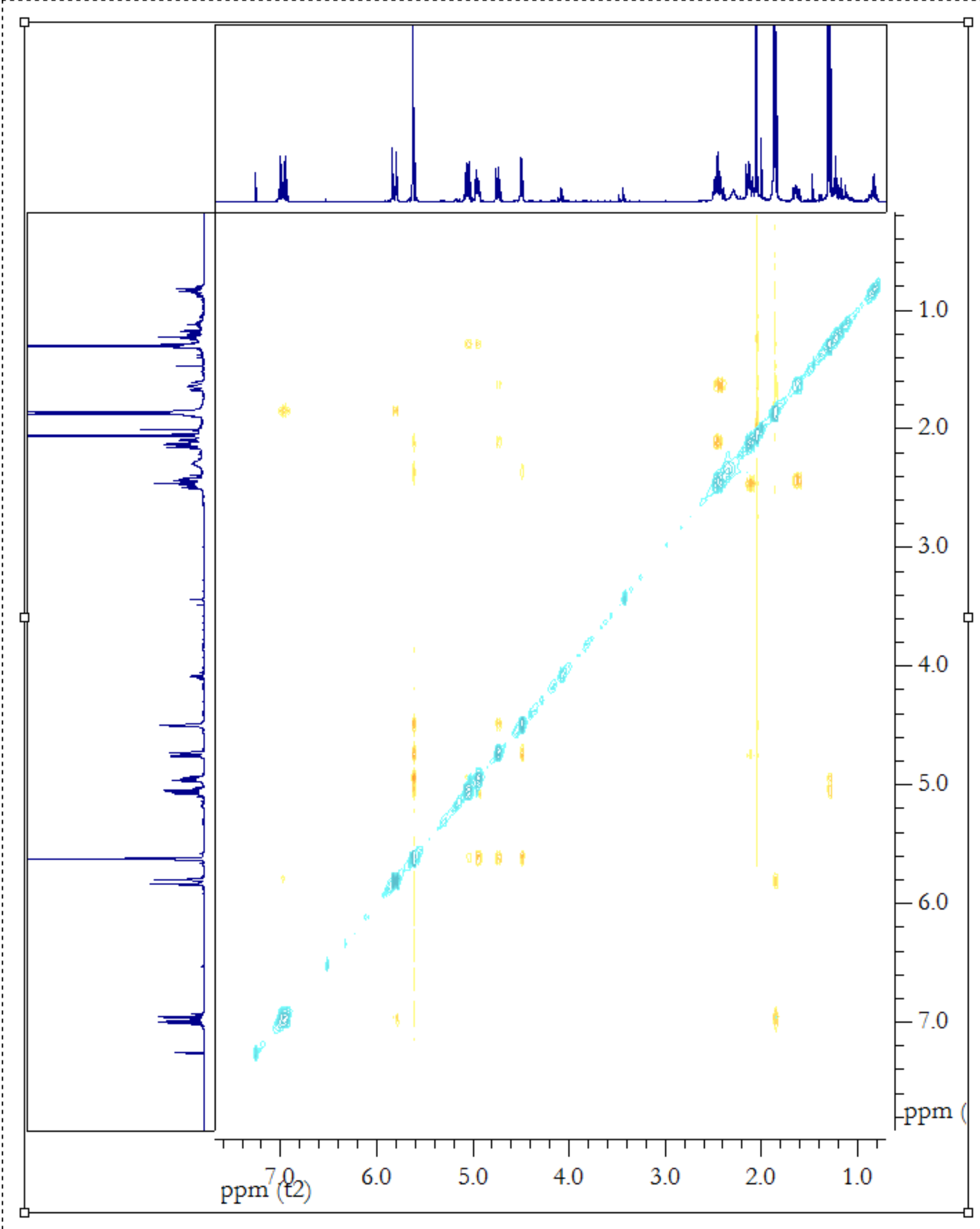


Figure S9. Mass spectrum (HRMS) of 1. (A - Full spectrum; B - Enlarged spectrum from 250 to $380 \mathrm{~m} / \mathrm{z}$ )

A

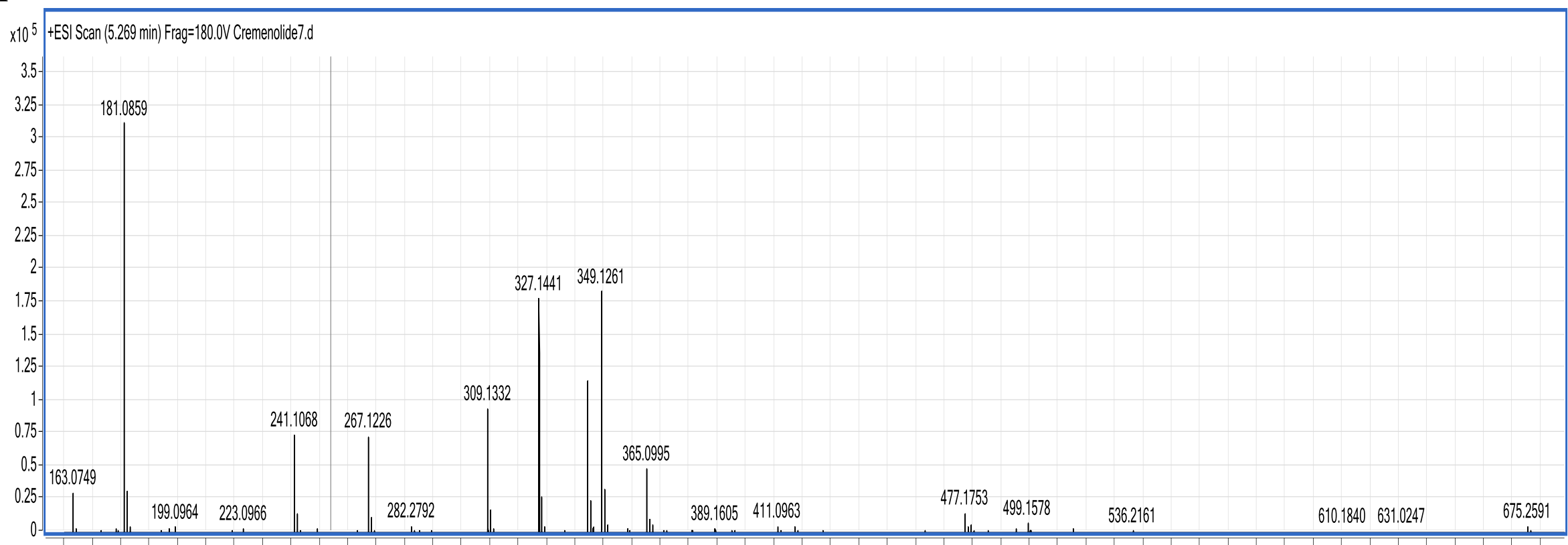

160170180190200210220230240250260270280290300310320330340350360370380390400410420430440450460470480490500510520530540550560570580590600610620630640650660670680 Counts vs. Mass-0-Charge $(\mathrm{m} / \mathrm{m})$ 


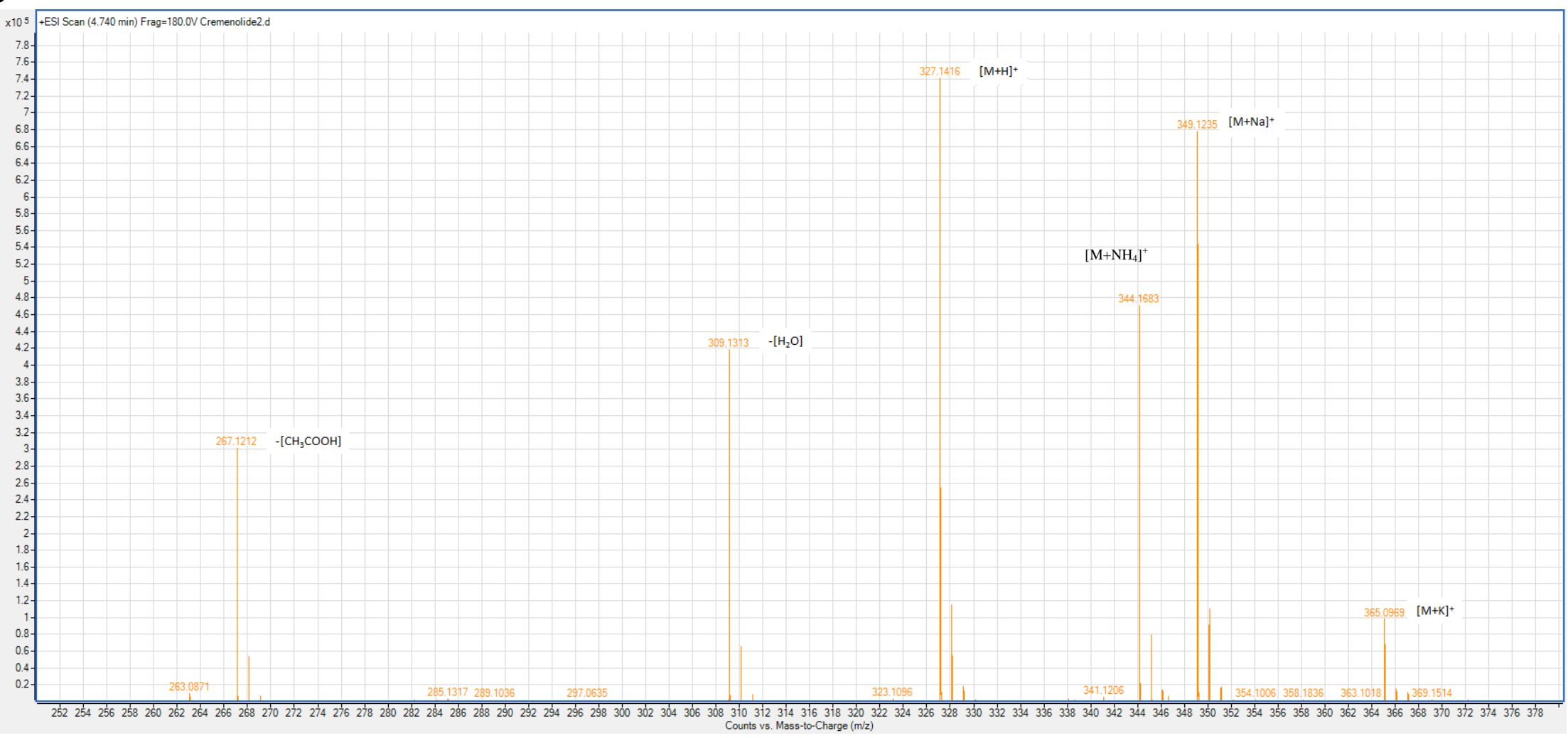


Figure S10. MS/MS of compound 1.

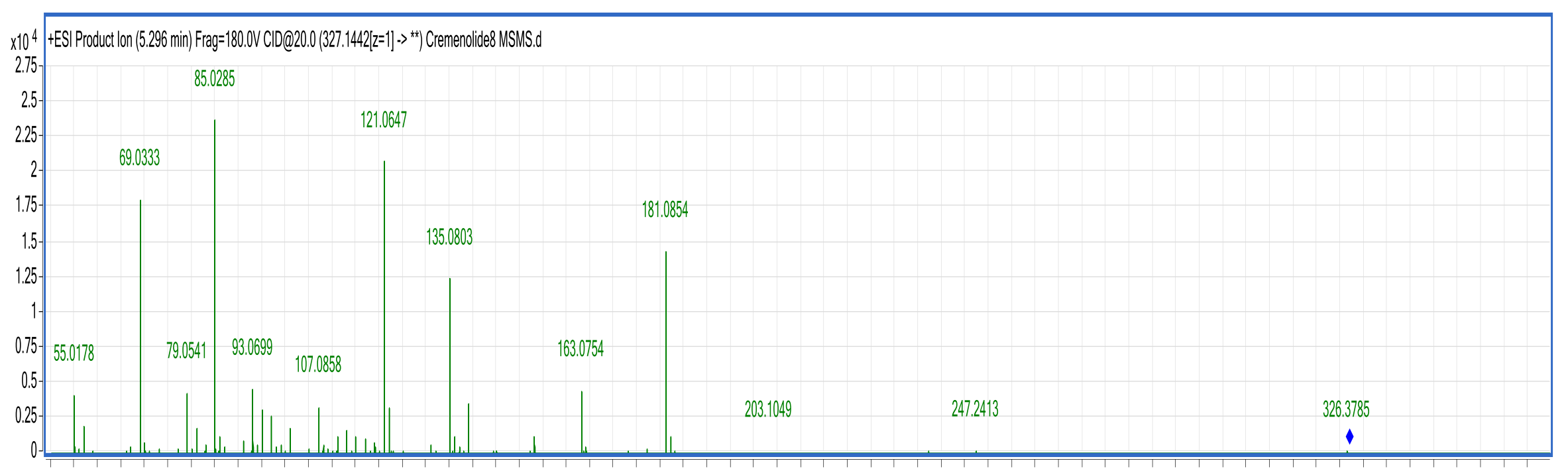

556065707580859095100105110115120125130135140145150155160165170175180185190195200205210215220225230235240245250255260265270275280285220225300305310315320325330335340345350355360365 Counts vs. Mass-0.-Charge $(m / 2)$ 


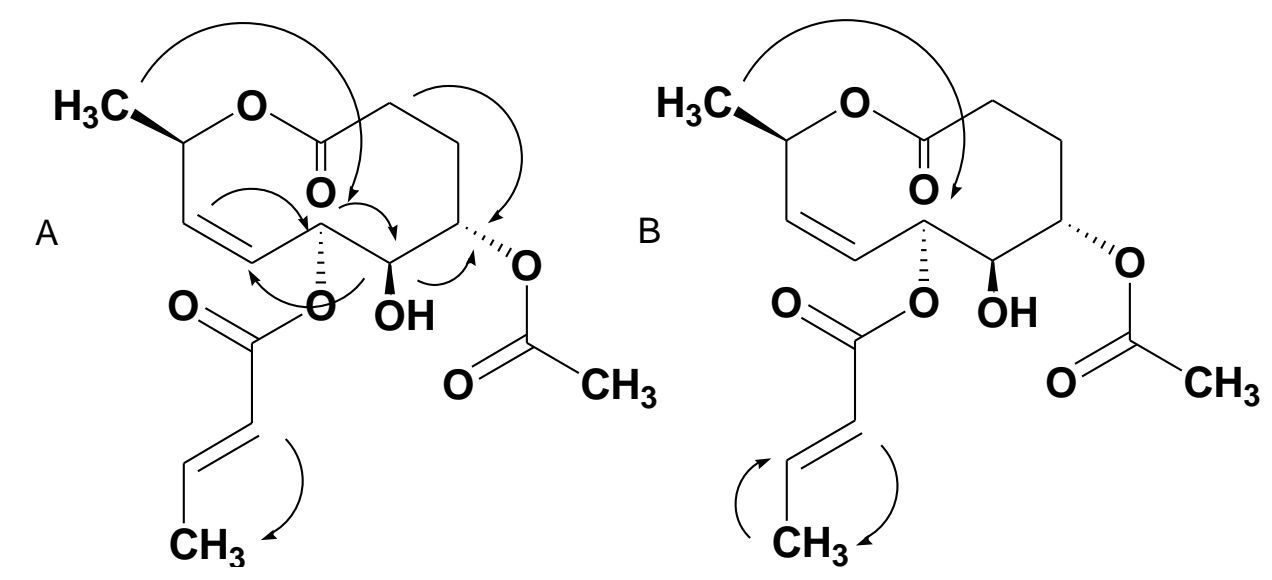

Figure S11. Determinant TOCSY (A) and NOESY (B) correlations detected for cremenolide (1) 
Table S1 ${ }^{1} \mathrm{H}$ and ${ }^{13} \mathrm{C}$ NMR spectral data of cremenolide (in $\mathrm{CDCl}_{3}$ ).

\begin{tabular}{|c|c|c|c|}
\hline \multicolumn{4}{|c|}{$\begin{array}{l}\text { Cremenolide (1). } \\
\text {. }\end{array}$} \\
\hline position & $\delta_{\mathrm{C}}$ mult. & $\delta_{\mathrm{H}}(\mathrm{J}$ in $\mathrm{Hz})$ & HMBC* \\
\hline 1 & $174.99 \mathrm{qC}$ & & $9,2 a, 2 b, 3 a, 3 b$ \\
\hline \multirow[t]{2}{*}{2} & $32.61 \mathrm{CH}_{2}$ & $2.46^{\mathrm{a}}$ & $3 a, 3 b, 4$ \\
\hline & & $2.13 \mathrm{~m}$ & \\
\hline \multirow[t]{2}{*}{3} & $23.86 \mathrm{CH}_{2}$ & $2.44^{\mathrm{a}}$ & $2 a, 4,5$ \\
\hline & & $1.64 \mathrm{~m}$ & \\
\hline 4 & $77.17 \mathrm{CH}$ & $4.74 \mathrm{dt}$ & $2 \mathrm{a}, 2 \mathrm{~b}, 3 \mathrm{a}, 3 \mathrm{~b}, 5,2$ "' \\
\hline 5 & $70.49 \mathrm{CH}$ & $4.5 \mathrm{~s}$ & $3 a, 3 b, 4,6,7$ \\
\hline 6 & $78.50 \mathrm{CH}$ & $4.96 \mathrm{~m}$ & $7,8,9,10$ \\
\hline 7 & $130.50 \mathrm{CH}$ & $5.62^{a}$ & $8,2^{\prime}, 6,5$ \\
\hline 8 & $128.56 \mathrm{CH}$ & $5.63 \mathrm{br} \mathrm{s}$ & $2^{\prime}, 7,6,5,9$ \\
\hline 9 & $71.63 \mathrm{CH}$ & $5.06 \mathrm{~m}$ & 10,8 \\
\hline 10 & $16.64 \mathrm{CH}_{3}$ & $1.3 \mathrm{~d}$ & 9,6 \\
\hline 1 ' & $165.38 \mathrm{qC}$ & & $6,2^{\prime}, 3^{\prime}, 4^{\prime}$ \\
\hline 2 & $122.01 \mathrm{CH}$ & $5.82 \mathrm{dd}(1.56,5.09)$ & $3^{\prime}, 4^{\prime}$ \\
\hline $3^{\prime}$ & $145.88 \mathrm{CH}$ & $6.9 \mathrm{dd}(1.55,7.05)$ & $2^{\prime}, 4^{\prime}$ \\
\hline 4, & $17.96 \mathrm{CH}_{3}$ & $1.86 \mathrm{dd}(7.05,7.4)$ & $2^{\prime}, 3^{\prime} 2^{\prime \prime}$ \\
\hline $1 "$ & $169.98 \mathrm{qC}$ & & $4,2 ”$ \\
\hline $2 "$ & $21.15 \mathrm{CH}_{3}$ & $2.06 \mathrm{~s}$ & $4,1^{\prime \prime}$ \\
\hline
\end{tabular}

*All correlations represent 2 or 3 bond couplings. ${ }^{\text {a }}$ overlapping NMR signals. Abbreviation, s: singlet, d: doublet, t: triplet, dd: doublet of doublets. 